Brief note

\title{
MECHANICAL PARAMETERS OF THE CURVILINEAR SQUEEZE FILM BEARING LUBRICATED BY A GECIM-WINER FLUID
}

\author{
A. WALICKA* and E. WALICKI \\ University of Zielona Góra, Faculty of Mechanical Engineering \\ ul. Szafrana 2, 65-516 Zielona Góra, POLAND \\ E-mails: A.Walicka@ijame.uz.zgora.pl,E.Walicki@ijame.uz.zgora.pl
}

\begin{abstract}
Based upon a Gecim-Winer fluid flow model, a curvilinear squeeze film bearing is considered. The equations of motion are given in a specific coordinates system. After general considerations on the Gecim-Winer fluid flow these equations are used to derive the Reynolds equation. The solution of this equation is obtained by a method of successive approximation. As a result one obtains formulae expressing the pressure distribution and load-carrying capacity. The numerical examples of the Gecim-Winer fluid flow in gaps of two simple bearings: radial and spherical are presented.
\end{abstract}

Key words: modified Reynolds equation, Gecim-Winer fluid, squeeze film, mechanical parameters of bearing, additives.

\section{Introduction}

Squeeze flow, a simplified model based on the Reynolds' lubrication theory, has been widely used in many applications, such as petroleum and chemical technology, food engineering, pharmaceutical manufacture, etc., involving a variety of fluids, including non-Newtonian fluids. Many investigators [1-5] have researched on the subject of squeeze flows. These flows are found in fabrication operations such as stamping, injection moulding, and sheet forming. Also, material properties of highly viscous fluids are measured with a device called the "plastometer" which incorporates a parallel-disk squeeze flow geometry $[1,6,7]$. In addition, such flows are encountered in lubrication systems, and there is a considerable interest as to the degree to which viscoplastic additives enhance the load-bearing capacity of lubricant.

The flows of Newtonian fluids in the clearance of a thrust bearing with impermeable surfaces have been examined theoretically. The bearing walls have been modelled as two disks, two conical or spherical surfaces. The more general case is established by the bearing formed by two surfaces of revolution [8].

The development of physico-chemistry has led to the introduction of new lubricants which enhance the conditions of the bearing operation and its performance. The mono and multigrade lubricants used in present-day tribological applications are blended with additives which are polymeric in nature. This blending results in a deviation of lubricant rheology from Newtonian to non-Newtonian.

Studies done on fluid film lubrication have employed several models such as the micropolar [8], couple-stress [9-12], viscoplastic [1-3, 7], pseudo-plastic [13-16] and mixture [17]. Naturally, this list is not complete and given only to present the possibility of mathematical modelling. A more complete list can be found in [9-11].

This paper is mainly concerned with the non-Newtonian effects in lubrication of the squeeze film bearing lubricated by a Gecim-Winer fluid (sometimes called a "tangent hyperbolic fluid") whose onedimensional model is given as follows [15]

\footnotetext{
* To whom correspondence should be addressed
} 


$$
\tau=\mu\left[\frac{\tanh (\beta \dot{\gamma})}{(\beta \dot{\gamma})}\right] \dot{\gamma}
$$

where $\tau$ is the shear stress, $\mu$ is the coefficient of viscosity, $\beta$ is the material constant, $\dot{\gamma}$ is the shear strain rate.

In what follows we will take into account only such values of $\beta$ for which the expression $(\beta \dot{\gamma})$ satisfies the relationship $[9,10]$

$$
(\beta \dot{\gamma})<1
$$

this can present the model Eq.(1.1) in the form of a series; limiting this series to the first two terms we have [9,10]

$$
\tau=\mu\left[1-\frac{(\beta \dot{\gamma})^{2}}{3}\right] \dot{\gamma}
$$

This formula will be used to model the Gecim-Winer fluid flow in a squeeze film bearing.

\section{Equations of motion of the Gecim-Winer fluid}

The three-dimensional form of the constitutive equation of the Gecim-Winer fluid is as follows $[9,10]$

$$
\boldsymbol{T}=-p \mathbf{1}+M \boldsymbol{A}_{l}, \quad M=\mu\left(1-\frac{\beta^{2}}{3} A^{2}\right), \quad A=\left[\frac{1}{2} \operatorname{tr}\left(\boldsymbol{A}_{l}^{2}\right)\right]^{1 / 2}
$$

where $\mathbf{1}$ is the unit tensor, $p$ is the pressure, $A$ is the second invariant of the stretching tensor $\boldsymbol{A}_{l}$ (the first Rivlin-Ericksen tensor) defined by

$$
\boldsymbol{A}_{l}=\boldsymbol{L}+\boldsymbol{L}^{T}, \quad \boldsymbol{L}=\operatorname{grad} \mathbf{v}
$$

and $\mathrm{v}$ is the velocity vector and $T$ denotes the transposition.

The general equations of motion of the Gecim-Winer fluid have the following forms:

- equation of continuity

$$
\operatorname{div} \mathbf{v}=0,
$$

- equation of momentum

$$
\rho \frac{d \mathbf{v}}{d t}=-\nabla p+\operatorname{div} \Lambda
$$

where

$$
\Lambda=M A_{l}
$$

Let us consider a thrust bearing with a curvilinear profile of the working surfaces shown in Fig.1. The lower fixed surface is described by the function $R(x)$ which denotes the radius of this surface. The bearing clearance thickness is given by the function $h(x, t)$. 

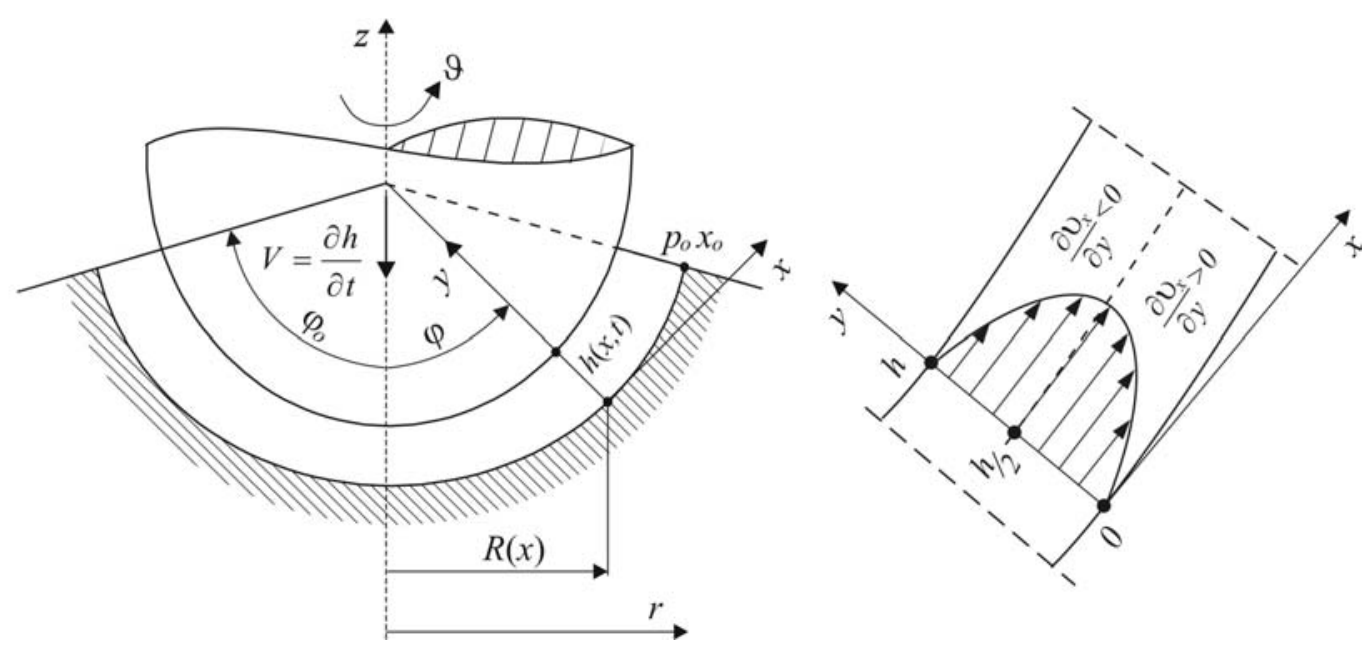

Fig.1. Geometry of a curvilinear bearing.

An intrinsic curvilinear orthogonal coordinate system $x, \vartheta, y$ linked with the lower bearing surface is also presented in Fig.1. The physical parameters of the Gecim-Winer fluid flow are the velocity components $v_{x}, v_{y}$, pressure $p$. With regard to the axial symmetry of the flow these parameters are not dependent on the angle $\vartheta$.

The assumptions typical for the flow in a narrow gap $[10,11]$ that

$$
h(x)<R(x) \quad v_{y}<v_{x}, \quad \frac{\partial}{\partial x}<<\frac{\partial}{\partial y}
$$

can be used to make order-of-magnitude arguments for Eqs (2.4)-(2.7).

If some asymptotic transformations are made, the same as in $[10,11]$ these equations can be reduced to a simpler form for slow flows

$$
\begin{aligned}
& \frac{1}{R} \frac{\partial\left(R v_{x}\right)}{\partial x}+\frac{\partial v_{y}}{\partial y}=0, \\
& \frac{1}{\mu} \frac{\partial p}{\partial x}=\frac{\partial^{2} v_{x}}{\partial y^{2}}-\beta^{2}\left|\frac{\partial v_{x}}{\partial y}\right|^{2} \frac{\partial^{2} v_{x}}{\partial y^{2}}, \\
& 0=-\frac{\partial p}{\partial y}
\end{aligned}
$$

The last equation results that

$$
p=p(x, t) \text {. }
$$

The problem statement is complete after specification of boundary conditions. These conditions for velocity component are stated as follows

$$
\begin{aligned}
& v_{x}(x, 0, t)=0, \quad v_{x}(x, h, t)=0, \\
& v_{y}(x, 0, t)=0, \quad v_{y}(x, h, t)=\frac{\partial h}{\partial t}=\dot{h} ;
\end{aligned}
$$

the boundary conditions for the pressure distribution will be presented in the next section. 


\section{Solution to the equations of motion}

Let us again consider the assumption (1.2) which can be presented in the form

$$
\beta^{2}\left|\frac{\partial v_{x}}{\partial y}\right|^{2}<1 \quad \text { or } \quad \beta^{2}=\delta<<1
$$

These relationships allow us to search for the solution of Eq.(2.7) in a series form

$$
v_{x}=\delta^{0} v_{0}+\delta^{l} v_{1}+\delta^{2} v_{2}
$$

Putting (3.2) into (2.7) and retaining only two first terms we find

$$
\frac{1}{\mu} \frac{\partial p}{\partial x}=\frac{\partial^{2} v_{0}}{\partial y^{2}}+\delta\left[\frac{\partial^{2} v_{1}}{\partial y^{2}}+\left|\frac{\partial v_{0}}{\partial y}\right|^{2} \frac{\partial^{2} v_{0}}{\partial y^{2}}\right]
$$

Solving this equation and taking into account the boundary conditions (2.10) we will obtain that

$$
\mathrm{v}_{x}=\left(-\frac{1}{2 \mu} \frac{\partial p}{\partial x}\right)\left(h y-y^{2}\right)-\frac{\beta^{2}}{24}\left[|h-2 y|^{4}-h^{4}\right]\left(-\frac{1}{2 \mu} \frac{\partial p}{\partial x}\right)^{3}
$$

To find the Reynolds equation it is necessary to introduce Eq.(3.4) into Eq.(2.6) and make the integration across the gap thickness. After carrying it we have

$$
\frac{1}{R} \frac{\partial}{\partial x} R h^{3}\left[\frac{\partial p}{\partial x}+\frac{\beta^{2} h^{2}}{16 \mu^{2}}\left(-\frac{\partial p}{\partial x}\right)^{3}\right]=12 \mu \frac{\partial h}{\partial t}
$$

the non-linear differential equation describing the pressure distribution.

Taking into account Eqs (3.1) we can search the solution of Eq.(3.5) in the form of a sum

$$
p=p^{(0)}+p^{(l)} .
$$

Assuming that $p^{(1)}>>p^{(0)}$ and substituting Eq.(3.6) into Eq.(3.5) we arrive at two linearized equations:

- the first one

$$
\frac{1}{R} \frac{\partial}{\partial x} R h^{3} \frac{\partial p}{\partial x}=12 \mu \frac{\partial h}{\partial t}
$$

- and the other

$$
\frac{1}{R} \frac{\partial}{\partial x} R h^{3} \frac{\partial p^{(1)}}{\partial x}=-\frac{\beta^{2}}{16 \mu^{2}} \frac{1}{R} \frac{\partial}{\partial x} R h^{5}\left(\frac{\partial p^{(0)}}{\partial x}\right)^{3} .
$$

The boundary conditions for the pressure in a squeeze film are 


$$
\left.\frac{\partial p^{(0)}}{\partial x}\right|_{x=0}=0, \quad p^{(0)}\left(x_{o}, t\right)=p_{o},\left.\quad \frac{\partial p^{(l)}}{\partial x}\right|_{x=0}=0, \quad p^{(l)}\left(x_{o}, t\right)=0
$$

The solutions of Eqs (3.7) and (3.8) are given, as follows

$$
p(x, t)=p_{o}-12 \mu\left[F_{o}-F(x, t)\right]
$$

where

$$
\begin{aligned}
& F(x, t)=I(x, t)+9 \beta^{2} J(x, t), \quad F_{o}=F\left(x_{o}, t\right), \\
& I(x, t)=\int \frac{\int R \dot{h} d x}{R h^{3}} d x, \quad J(x, t)=\int \frac{\left(\int R \dot{h} d x\right)^{3}}{R^{3} h^{7}} d x .
\end{aligned}
$$

The load-carrying capacity is defined, by

$$
N=2 \pi \int_{0}^{x_{o}}\left(p-p_{o}\right) R \cos \varphi d x
$$

the sense of the angle $\varphi$ arises from Fig.1.

\section{Examples of squeeze film bearings}

Let us consider a radial thrust bearing with squeezed film of the Gecim-Winer lubricant modelled by two parallel disks (Fig.2).

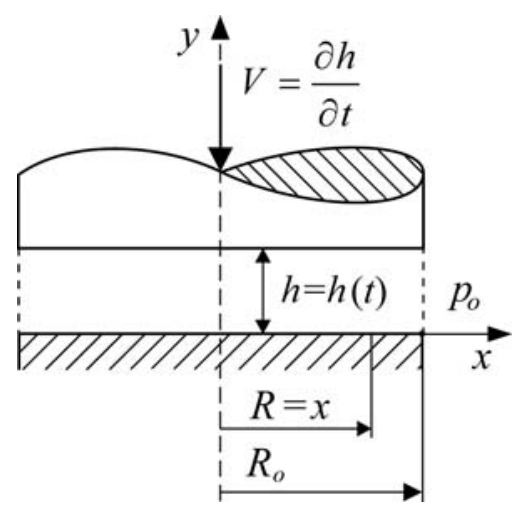

$$
\begin{aligned}
& R=x, \quad \tilde{x}=\frac{x}{x_{o}}, \quad \tilde{h}=\frac{h}{h_{o}}=e(t), \\
& e(t)=1-\varepsilon(t), \quad \dot{\varepsilon}=\frac{d \varepsilon}{d t},
\end{aligned}
$$

Fig.2. Squeeze film in a radial thrust bearing.

Introducing the following parameters

$$
\tilde{p}=\frac{\left(p-p_{o}\right)}{\mu \dot{\varepsilon}}\left(\frac{h_{o}}{x_{o}}\right)^{2}, \quad \tilde{N}=\frac{N}{\mu \dot{\varepsilon} x_{o}^{2}}\left(\frac{h_{o}}{x_{o}}\right)^{2}, \quad \lambda=\beta^{2}\left(\frac{\dot{\varepsilon} x_{o}}{h_{o}}\right)^{2}
$$


we will obtain the formulae for the dimensionless pressure distribution and load-carrying capacity for the radial thrust bearing with a squeeze film of the lubricant

$$
\tilde{p}=\frac{3}{e^{3}}\left[1-\tilde{x}^{2}-\frac{9}{8} \frac{\lambda}{e^{4}}\left(1-\tilde{x}^{4}\right)\right], \quad \tilde{N}=\frac{3 \pi}{2 e^{3}}\left(1-\frac{3 \lambda}{2 e^{4}}\right)
$$

Plots of the dimensionless pressure distribution and load-carrying capacity are presented in Figs 3 and 4.

a)

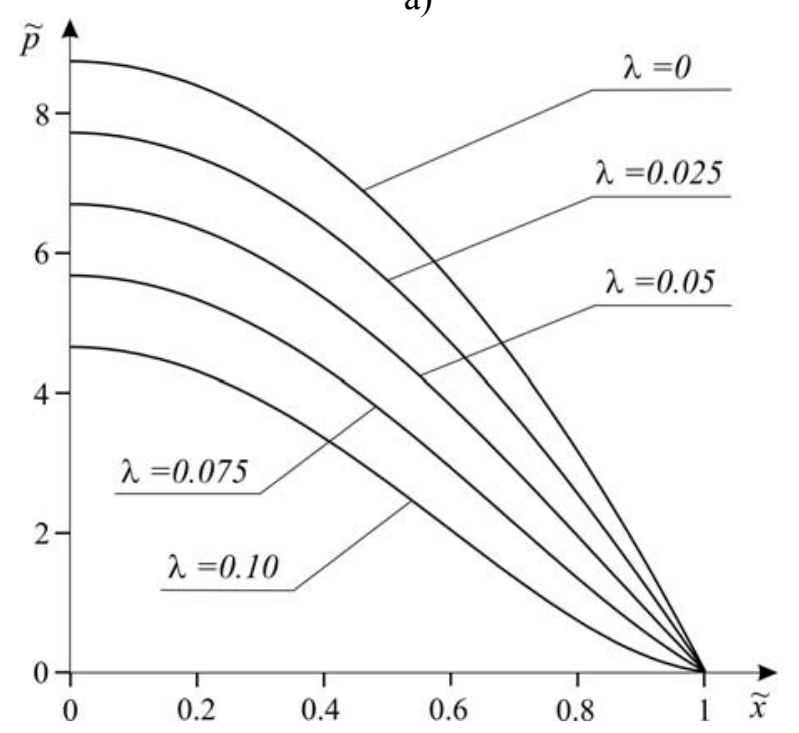

b)

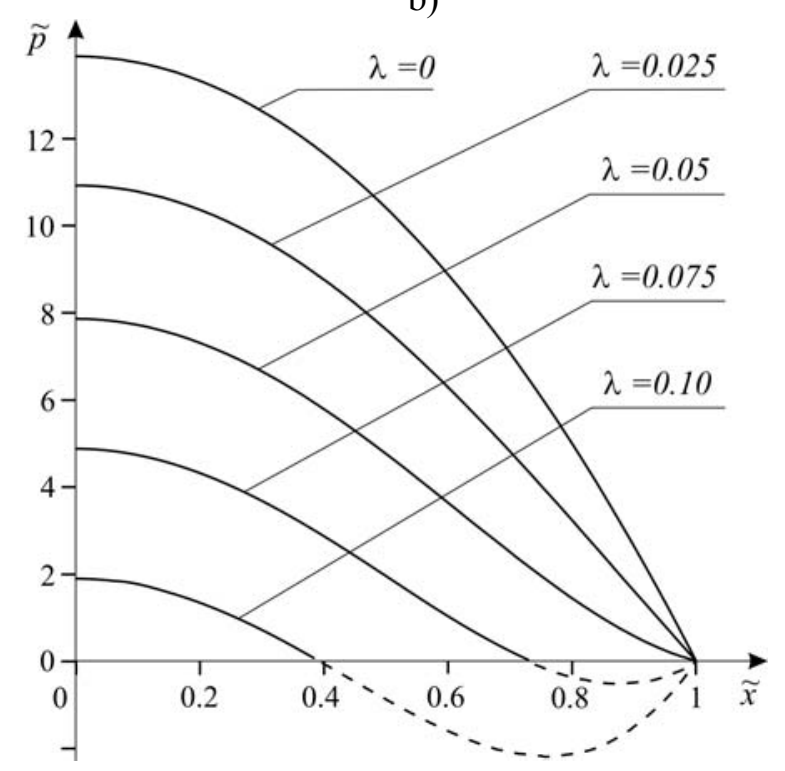

Fig.3. Dimensionless pressure distribution in the radial squeeze film bearing for different values of $\lambda$ and for: a) $\varepsilon=0.3$ and b) $\varepsilon=0.4$

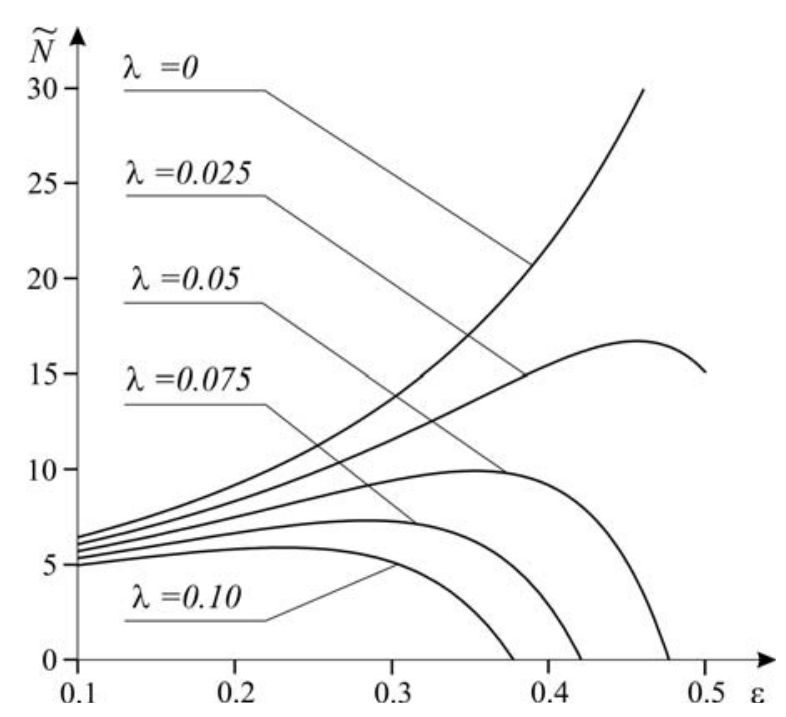

Fig.4. Dimensionless load-carrying capacity in the radial squeeze film bearing for different values of $\lambda$.

Let us consider now a spherical squeeze film bearing shown in Fig. 5. 
Introducing the following parameters:

$$
\tilde{p}=\frac{\left(p-p_{o}\right)}{\mu \dot{\varepsilon}}\left(\frac{C}{R_{r}}\right)^{2}, \quad \tilde{N}=\frac{N C^{2}}{\mu \dot{\varepsilon} R_{r}^{4}}, \quad \lambda=\beta^{2}\left(\frac{\dot{\varepsilon} R_{r}}{C}\right)^{2},
$$

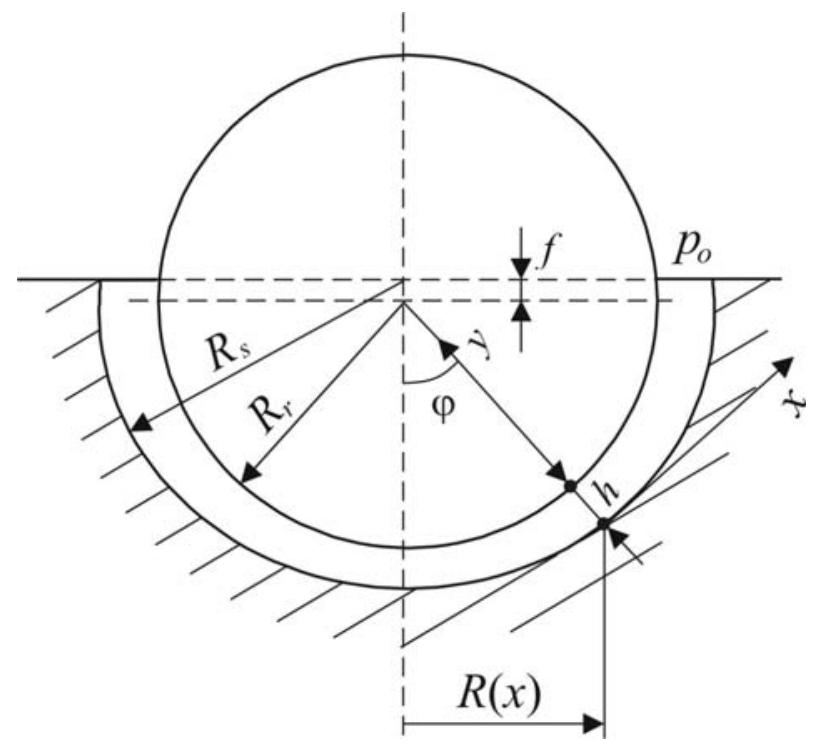

$$
\begin{aligned}
& C=R_{s}-R_{r}, \quad \varepsilon(t)=\frac{f}{C}, \\
& \varphi=\frac{x}{R_{r}}, \quad R=R_{s} \sin \varphi, \\
& u=\tilde{h}=\frac{h}{C}=1-\varepsilon \cos \varphi, \\
& u_{o}=1-\varepsilon \cos \varphi_{o}, \quad e=1-\varepsilon .
\end{aligned}
$$

Fig.5. Spherical squeeze film bearing ( for $\varphi_{o}=0.5 \pi$ ).

we will obtain the formulae for the dimensionless pressure distribution and load-carrying capacity for the spherical squeeze film bearing:

$$
\begin{aligned}
& \tilde{p}=-\frac{3}{\varepsilon}\left\{\left(\frac{1}{u_{o}^{2}}-\frac{1}{u^{2}}\right)+\frac{3}{4} \frac{\lambda}{\varepsilon^{2}}\left[\left(1-\varepsilon^{2}\right)\left(\frac{1}{u_{o}^{6}}-\frac{1}{u^{6}}\right)+\right.\right. \\
& \left.\left.-\frac{12}{5}\left(\frac{1}{u_{o}^{5}}-\frac{1}{u^{5}}\right)+\frac{3}{2}\left(\frac{1}{u_{o}^{4}}-\frac{1}{u^{4}}\right)\right]\right\}, \\
& \tilde{N}=-\frac{6 \pi}{\varepsilon^{3}}\left(\ln u_{o}-\ln e+\frac{1}{u_{o}}-\frac{1}{e}-\frac{\Phi}{2 u_{o}^{2}}+\frac{3}{4} \frac{\lambda}{\varepsilon^{2}}\left\{( 1 - \varepsilon ^ { 2 } ) \left[\frac{1}{5}\left(\frac{1}{u_{o}^{5}}-\frac{1}{e^{5}}\right)+\right.\right.\right. \\
& \left.-\frac{1}{4}\left(\frac{1}{u_{o}^{4}}-\frac{1}{e^{4}}\right)-\frac{\Phi}{2 u_{o}^{6}}\right]-\frac{12}{5}\left[\frac{1}{4}\left(\frac{1}{u_{o}^{4}}-\frac{1}{e^{4}}\right)-\frac{1}{3}\left(\frac{1}{u_{o}^{3}}-\frac{1}{e^{3}}\right)-\frac{\Phi}{2 u_{o}^{5}}\right]+ \\
& \left.\left.+\frac{3}{2}\left[\frac{1}{3}\left(\frac{1}{u_{o}^{3}}-\frac{1}{e^{3}}\right)-\frac{1}{2}\left(\frac{1}{u_{o}^{2}}-\frac{1}{e^{2}}\right)-\frac{\Phi}{2 u_{o}^{4}}\right]\right\}\right) ; \quad \Phi=u_{o}^{2}-e^{2}-2\left(u_{o}-e\right) .
\end{aligned}
$$
and 7.

Plots of the dimensionless pressure distribution and load-carrying capacity are presented in Figs 6 
a)

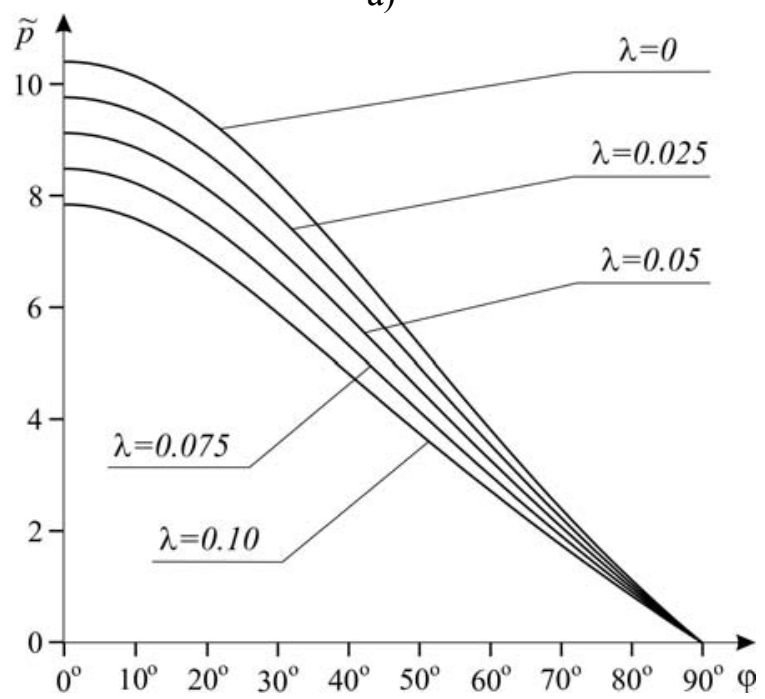

b)

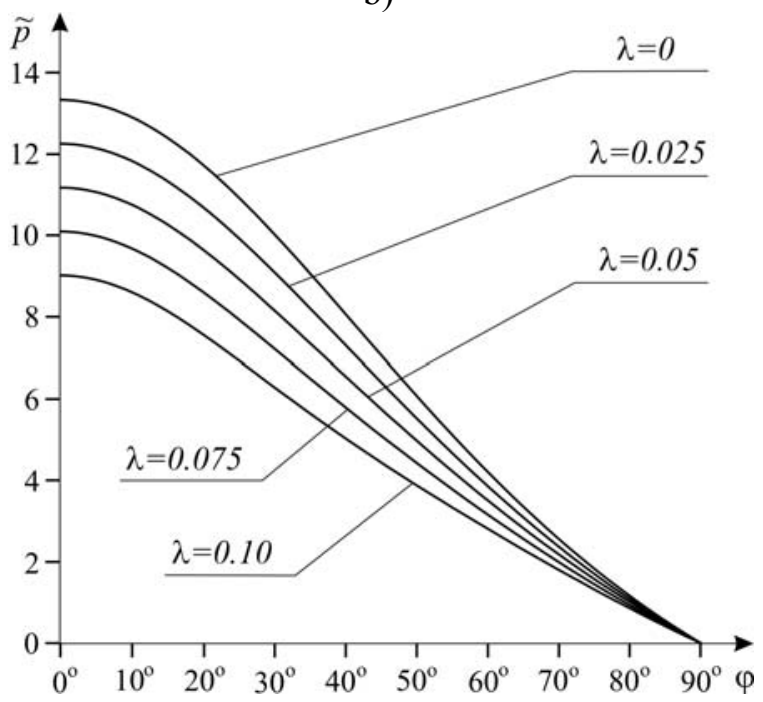

Fig. 6. Dimensionless pressure distribution in the spherical squeeze film bearing for different values of $\lambda$ and for: a) $\varepsilon=0.3$ and b) $\varepsilon=0.4$.

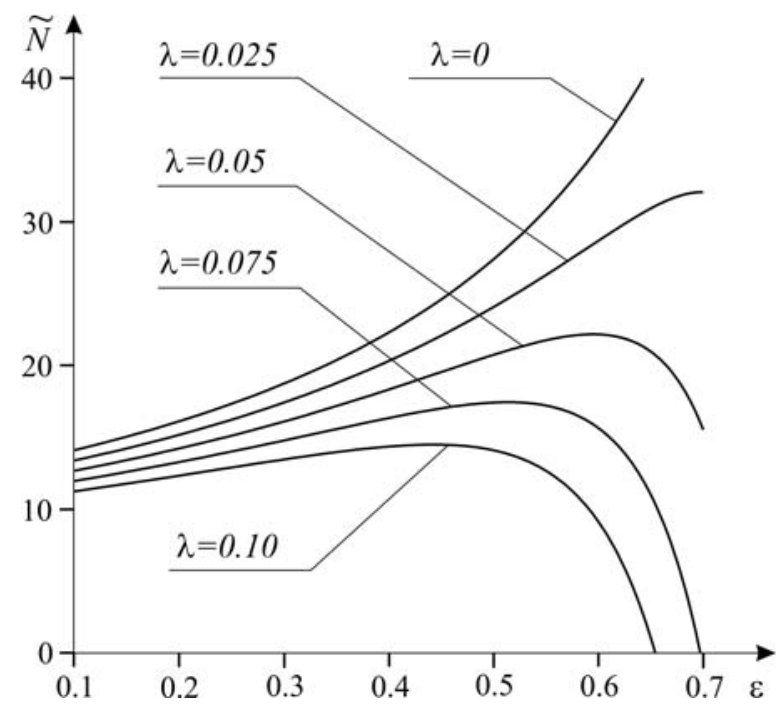

Fig.7. Dimensionless load-carrying capacity in the spherical squeeze film bearing for different values of $\lambda$.

\section{Conclusion}

The modified Reynolds equation for a Gecim-Winer type of a pseudo-plastic lubricant flowing in the gap of a thrust curvilinear bearing with squeeze film is derived. As a result the general formulae for pressure distribution and load-carrying capacity are obtained. It follows from the calculations carried out and their graphic presentations that the both magnitudes are dependent on the values of rheological parameters $\beta$ or $\lambda$. It may be concluded that in both the bearings under considerations the pressures and load-carrying capacities decrease with the increase of $\lambda$.

These decreases are more visible for the radial squeeze film bearing. The pressure distribution for the radial squeeze film bearing is strongly dependent on the values of the squeezing ratio $\varepsilon$ and dimensionless coefficient of pseudo-plasticity $\lambda$. With the increase of the both parameters the pressure quickly decreases until 
the appearance of cavitation; this phenomenon is marked on the pressure plots (see Fig. 3.b) by dashed lines. The carrying-capacity for this bearing also quickly decreases with the increase of $\varepsilon$ and $\lambda$ with respect to the case of the Newtonian lubricant $(\lambda=0)$.

The pressure distribution and carrying-capacity for the spherical squeeze film bearing also are dependent on the values of $\varepsilon$ and $\lambda$ but this dependence is weaker than that for the radial bearing. Here the cavitation may also appear, but for larger values of $\varepsilon$ and $\lambda$.

\section{References}

[1] Covey G.H. and Stanmore B.R. (1981): Use of the parallel-plate plastometer for the characterisation of viscous fluids with a yield stress. - J. Non-Newtonian Fluid Mech, vol.8, pp.249-260.

[2] Dai G. and Bird R.B. (1981): Radial flow of Bingham fluid between two fixed circular disks. - J. Non-Newtonian Fluid Mech., vol.8, pp.349-355.

[3] Lipscomb C.C. and Denn M.M. (1984): Flow of Bingham fluids in complex geometries. - J. Non-Newtonian Fluid Mech., vol.14, pp.337-349.

[4] Adams M.J. and Edmondson B. (1987): Forces between particles in continuous and discrete liquid media. - In: B.J.Briscoe and M.J.Adams (Eds): Tribology in particulate technology, pp.154-172, IOP Publishing, New York.

[5] Rodin G.J. (1996): Squeeze film between two spheres in a power-law fluid. - J.Non-Newtonian Fluid Mech., vol.63, pp.141-152.

[6] Engman J., Servais C. and Burbidge A.S. (2005): Squeeze flow theory and applications to rheometry: a review. J. Non-Newtonian Fluid Mech., pp.132:1-27.

[7] Xu C., Yuan L., Xu Y. and Hang W. (2010): Squeeze flow of interstitial Herschel-Bulkley fluid between two rigid spheres. - Particuology, vol.8, pp.360-364.

[8] Walicka A. (1974): Micropolar Flow in a Slot Between Rotating Surfaces of Revolution. - Zielona Góra: TU Press.

[9] Walicka A. (2002): Rheodynamics of Non-Newtonian Fluids Flow in Straight and Curved Channels (in Polish). -Zielona Gora: University Press.

[10] Walicka A. (2002): Rotational Flows of Rheological Complex Media in Narrow Annular Channels (in Russian). - Zielona Góra: University Press, 2002.

[11] Walicki E. (2005): Rheodynamics of Slide Bearings Lubrication (in Polish). -, Zielona Gora: University Press.

[12] Jurczak P., Walicka A., Walicki E. and Michalski D. (2006): Flow of a couple-stress fluid in a curvilinear thrust bearing with one porous pad. - Tribologia vol.3, pp.63-75.

[13] Swamy S.T.N., Prabhu B.S. and Rao B.V.A. (1975): Stiffness and damping characteristics of finite width journal bearing with a non-Newtonian film and their application to instability prediction. - Wear, vol.32, pp.379-390.

[14] Rajalingham C., Rao B.V.A. and Prabu S. (1978): The effect of a non-Newtonian lubricant on piston ring lubrication. - Wear, vol.50, pp.47-57.

[15] Gecim B. and Winer W.O. (1980): Lubricant limiting shear stress effect on EHD film thickness. - J. Lubric. Trans. ASME, vol.102, No2, pp.213-221.

[16] Walicka A. and Walicki E. (2016): Mechanical parameters of the squeeze film curvilinear bearing lubricated by a Prandtl fluid. - Int. J. Appl. Mech. Enging, vol.21. No4, pp.967-977.

[17] Khonsari M.M. and Dai F. (1972): On the mixture flow problem in lubrication of hydrodynamic bearing: small solid volume fraction. - STLE Trib. Trans., vol.35, No1, pp.45-52. 